OPEN ACCESS

Edited by:

Qiang Xu,

Nanyang Technological

University, Singapore

Reviewed by:

Xiaowen Zhang,

Guilin University of Electronic Technology, China

Zhijie Luo,

Zhongkai University of Agriculture and Engineering, China

*Correspondence:

Wei L

wei.li@guohua-oet.com

Specialty section:

This article was submitted to

Optics and Photonics,

a section of the journal

Frontiers in Physics

Received: 19 January 2021

Accepted: 22 February 2021

Published: 16 March 2021

Citation:

Wang L, Zhang H, Li W, Li J, Yi Z, Wan $Q$, Zhang J and Ma P (2021)

Driving Scheme Optimization for Electrowetting Displays Based on

Contact Angle Hysteresis to Achieve Precise Gray-Scales.

Front. Phys. 9:655547.

doi: 10.3389/fphy.2021.655547

\section{Driving Scheme Optimization for Electrowetting Displays Based on Contact Angle Hysteresis to Achieve Precise Gray-Scales}

\author{
Li Wang ${ }^{1}$, Hu Zhang ${ }^{2}$, Wei $\mathrm{Li}^{3 *}$, Jiaxuan $\mathrm{Li}^{4}$, Zhenyu $\mathrm{Yi}^{4}{ }^{4}$, Qiming Wan ${ }^{1}$, Jitao Zhang ${ }^{1}$ and \\ Pengchang $\mathrm{Ma}^{1}$
}

${ }^{1}$ School of Information Engineering, Zhongshan Polytechnic, Zhongshan, China, ${ }^{2}$ School of Electronic Science and Engineering (National Exemplary School of Microelectronics), University of Electronic Science and Technology of China, Chengdu, China, ${ }^{3}$ South China Academy of Advanced Optoelectronics, South China Normal University, Guangzhou, China, ${ }^{4}$ College of Electron and Information, Zhongshan Institute, University of Electronic Science and Technology of China, Zhongshan, China

As a new display technology, electrowetting display (EWD) has many excellent display characteristics, such as paper-like, low power consumption, quick response and full color. These characteristics make EWD devices very suitable for portable devices. However, the gray-scale distortion caused by the contact angle hysteresis of EWDs seriously affects the accuracy of gray-scale display. To improve this phenomenon, the hysteresis curve of an EWD panel was studied according to the motion characteristics of advancing contact angle and receding contact angle of oil in a pixel. Then, a driving scheme for EWDs using alternating current (AC) voltage instead of direct current (DC) voltage was proposed in this paper. And the advantages and disadvantages of the driving scheme at different $\mathrm{AC}$ frequencies from 90 to $2,700 \mathrm{~Hz}$ were analyzed through experiments. According to the stability of aperture ratio in EWDs, a $470 \mathrm{~Hz} \mathrm{AC}$ driving scheme was determined. Experimental results showed that the aperture ratio distortion of EWDs could be reduced from 35.82 to $5.97 \%$, which significantly improved the display performance of pixel units.

\footnotetext{
Keywords: electrowetting display, contact angle hysteresis, alternating current voltage, driving scheme, aperture ratio
}

\section{INTRODUCTION}

Electrowetting display (EWD) is a new type of electronic paper display technology with excellent display characteristics: (a) reflective display, paper-like reading experience [1]; (b) response speed of several milliseconds which can meet the needs of video playback [2]; (c) paper-like reflective display mode, with low power consumption [3]; (d) high reflectivity and true color display can be implemented [4]; (e) pixel structure can be attached to flexible backplanes [5]; (f) high degree of overlap with the existing liquid crystal display (LCD) manufacturing process, which means a low manufacturing cost [6]. These advantages make EWD technology to be known as the most promising next-generation paper-like display technology.

The EWD technology was first proposed by Beni and Hackwood [7]. In 2003, Hayes proposed a fast display EWD scheme, which could realize a simple video playback function [8]. However, 
when EWDs were driven, the phenomenon of contact angle hysteresis would occur, which had a bad effect on the grayscale display performance of pixels in EWDs $[9,10]$. The linear inconsistency of advancing contact angle and receding contact angle increased the complexity for controlling gray-scales [11]. In the process of pixel gray-scales display, it is difficult to control the gray-scale accurately, and gray-scale distortion may be caused. Therefore, it is necessary to analyze the influence of contact angle hysteresis and study how to optimize the contact angle hysteresis in EWDs.

Many scholars have studied the hysteresis phenomenon of EWDs. An AC voltage driving scheme was proposed by studying the balance among the surface tension of oil wetting, pinning force and electrostatic force on the contact line. It was proved that the hysteresis of the contact angle of a moving droplet could be reduced during a wetting process [12]. However, researchers did not further explore parameters of the AC voltage. Then, according to pixel characteristics, two driving methods of using hydrophilic patches or adding steps for pixels were proposed to enhance the motion control of oil and realize a hysteresis-free gray-scale conversion of pixels [13]. However, the transparent electrode of the pixel could be blocked by hydrophilic patches and the increased steps, which could reduce the reflectivity of a pixel. And then, a driving waveform with an optimized voltage slope was proposed, this method can improve the aperture ratio and reduce the hysteresis of pixels [14]. Although the PWM (pulse width modulation) could compensate for the hysteresis to a certain extent, it means an increase of response time, a decrease of effective frames, and an increase of power consumption [15]. At the same time, the PWM can only be used in fully opened and completely closed for driving a pixel, and the display ability of EWDs is limited by this feature.

In this paper, the influence of contact angle hysteresis on the gray-scale was analyzed when the driving voltage was turned on and off, and an AC voltage driving scheme was proposed, the influence of contact angle hysteresis on display performance could be significantly reduced by the proposed AC driving scheme compared with the traditional DC voltage driving scheme.

\section{PRINCIPLES OF SYSTEM DESIGN Display Principle of EWDs}

Wettability is great significance for EWDs. Wettability describes the relationship between the contact angle and the surface tension of droplets. The surface tension can be expressed by equation (1), $\gamma_{L G}$ is a liquid-gas surface tension, $f$ is the frequency of the surface wave, $\lambda$ is the wavelength of the surface wave, $\rho$ is the density of droplets. The relationship between contact angle and surface tension can be expressed by equation (2) [16], $\theta_{0}$ is a static contact angle, $\gamma_{S G}$ is a solid-gas surface tension and $\gamma_{S L}$ is a solid-liquid surface tension.

$$
\begin{aligned}
\gamma_{L G} & =\frac{f^{2} \lambda^{3} \rho}{2 \pi} \\
\cos \theta_{0} & =\left(\gamma_{S G}-\gamma_{S L}\right) \frac{2 \pi}{f^{2} \lambda^{3} \rho}
\end{aligned}
$$

Lippmann-Young equation was first proposed by Gabriel Lippmann in 1898, and it is regarded as the theoretical foundation of electrowetting. This equation describes the changing process of the contact angle of droplets on the solid surface with an applied voltage. As shown in equation (3) [17]:

$$
\cos \theta=\frac{\gamma_{s e}-\gamma_{s o}}{\gamma_{e o}}+\frac{1}{2} \frac{\varepsilon_{0} \varepsilon_{r}}{d \gamma_{e o}} V^{2}
$$

Where, $\theta$ is the contact angle when driving voltage is $V$, $\varepsilon_{0}$ is the vacuum dielectric constant, $\varepsilon_{r}$ is the relative dielectric constant, $d$ is the thickness of a hydrophobic insulation layer, $\gamma_{e o}$ is the interface tension between oil and electrolyte solution, and $V$ is the driving voltage. As shown in Figure 1, The other two interfacial tensions are $\gamma_{s o}$ and $\gamma_{s e}, \gamma_{s o}$ is the interfacial tension between the hydrophobic insulation layer and oil. $\gamma_{s e}$ is the interfacial tension between the hydrophobic insulation layer and electrolyte solution. When the driving voltage is $0 \mathrm{~V}$, the non-polar oil is laid on the hydrophobic insulation layer, and the contact angle is small, close to zero.

EWD is an optical switch for realizing gray-scale display by controlling the movement of colored oil by applying voltage. In practical application, the display is realized by an optical stack. It consists of a white substrate, a hydrophobic insulation layer, colored oil, water and an ITO (indium tin oxide) glass. When the voltage is turned off, the force among the colored oil, the hydrophobic insulation layer and the water is balanced. At this time, $\gamma_{e o}>\gamma_{s o}$, and contact angle $\theta<90$. The colored oil is laid between the water and the hydrophobic insulation, which can show a hydrophilicity and become a continuous spreading film. And we could see the color of oil, as shown in Figure 2A, the state is in the lowest energy state at this time.

Next, the voltage is connected to the upper and lower plates of ITO. As shown in Figure 2B, according to Lippmann-Young equation, with the increase of electric field, the surface tension among insulation layer, oil film and water can be increased, and the indirect antennae of the interface become larger. The original balance can be broken by the electric field force generated by the voltage difference. Water instead of the oil to contact with the hydrophobic insulation layer surface. At this time, $\gamma_{e o}<\gamma_{s o}$, and contact angle $\theta>90$. The oil is pushed to a corner of pixels, so pixels show color of the white substate. The colored oil shows hydrophobicity.

\section{Principle of Contact Angle Hysteresis}

In an EWD driving process, as the driving voltage increases from $0 V$ to the maximum voltage $V_{m}$, the oil in the pixel is gradually gathered. When the driving voltage reaches the maximum value, the oil can be completely pushed to a corner. In this process, the higher the voltage, the larger the contact angle. The process of increasing voltage is the advancing process, and the contact angle is also called as advancing angle. On the contrary, the process of reducing the voltage from $V_{m}$ to $0 \mathrm{~V}$ is called as receding process, and the contact angle at this time is also called as the receding angle. With a same driving voltage value, the difference between the advancing angle and the receding angle is the contact angle hysteresis in EWDs. 


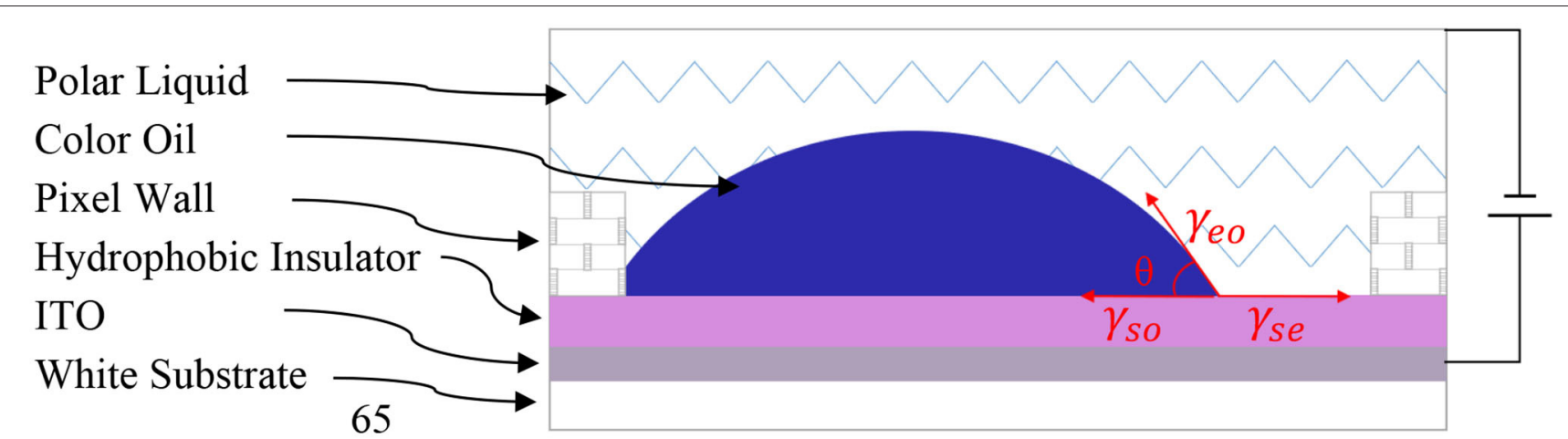

FIGURE 1 | The structure of an EWD pixel. The colored oil was pushed to a corner of the pixel by driving voltage. The pixel would display the color of oil and part of the white substrate.

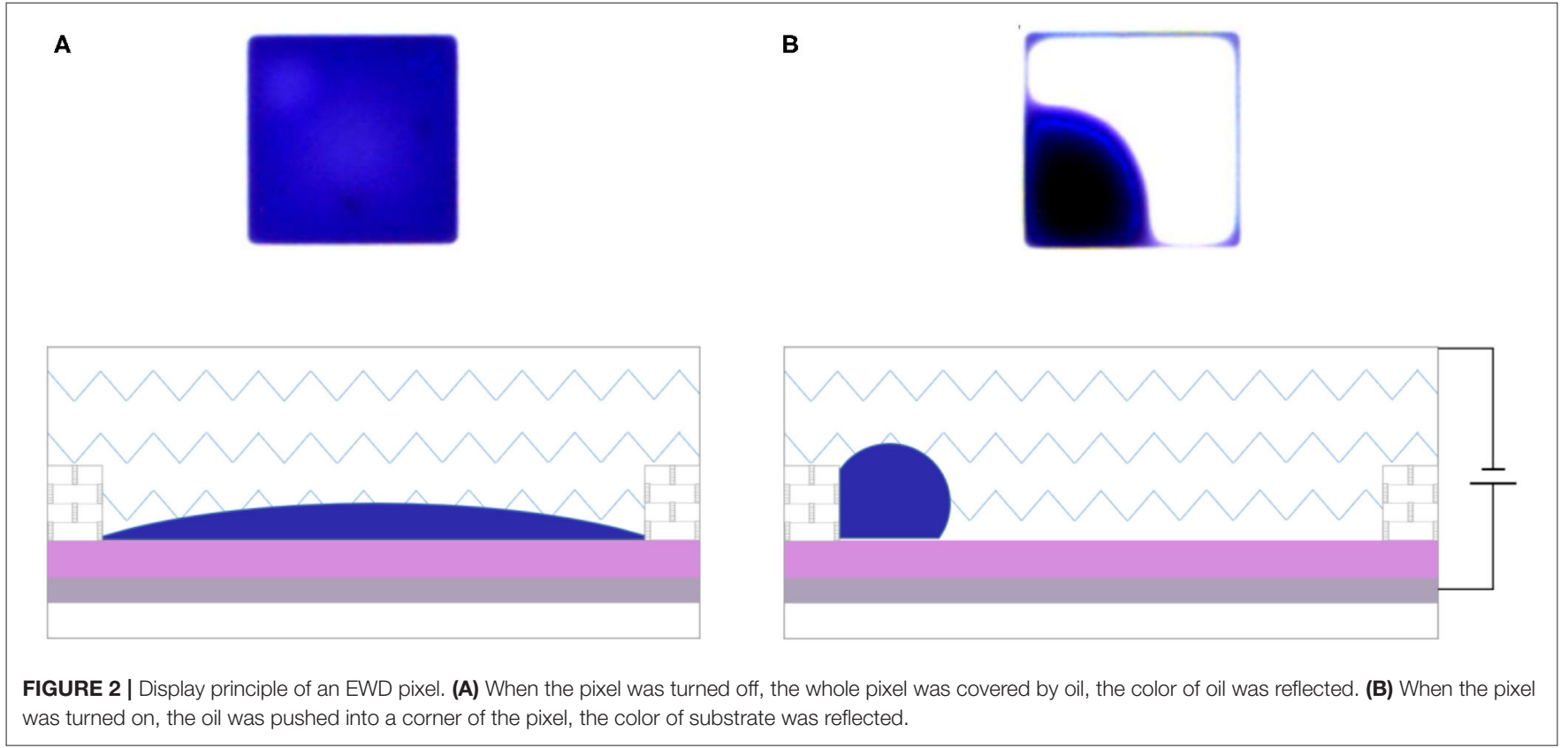

The aperture ratio is a proportion of the exposed white substrate area in a whole pixel. The different aperture ratio represents different optical states. The surface area of oil can be expressed as equation (4), and the aperture ratio can be defined as equation (5) [18].

$$
\begin{aligned}
S_{\text {oil }} & =\pi \sin ^{2} \theta\left(\frac{B_{\text {oil }}}{\frac{2}{3} \pi\left(1-\frac{3}{2} \cos \theta+\frac{1}{2} \cos ^{3} \theta\right)}\right)^{\frac{2}{3}} \\
W_{A}(V) & =\left(1-\frac{\pi \sin ^{2} \theta B_{\text {oil }} \frac{2}{3}}{S_{\text {pix }}\left(\frac{2}{3} \pi\left(1-\frac{3}{2} \cos \theta+\frac{1}{2} \cos ^{3} \theta\right)\right)^{\frac{2}{3}}}\right) \times 100 \%
\end{aligned}
$$

Where, $W_{A}(V)$ is the aperture ratio, $S_{\text {oil }}$ represents the surface area of oil when voltage $V$ is applied to EWDs. $S_{\text {pix }}$ is the surface area of a whole pixel, and $B_{o i l}$ is the volume of the oil. The pixel wall can divide an EWD into several pixels. This transparent grid structure has a small area which can be ignored in calculating the aperture ratio.

\section{EXPERIMENTAL RESULTS AND DISCUSSION}

\section{Experimental Platform and Experimental Materials}

In order to measure the display state of pixels, a complete experimental platform was built. As shown in Figure 3, the experimental equipment mainly includes: (a) is a GDS-3354 oscilloscope (Shanghai GuWei Electronics Co., Ltd, Shanghai, China); (b) is an AFG3052C function generator, produced by Tektronix (United States); (c) is an ATA-2022H amplifier, produced by Agitek (Xi'an, China); (d) is a XTL-165 microscope, produced by Phenix (Jiangxi, China), and (e) is a computer. The experimental process was described as follows: First, the required waveform was edited by the computer. Second, the 


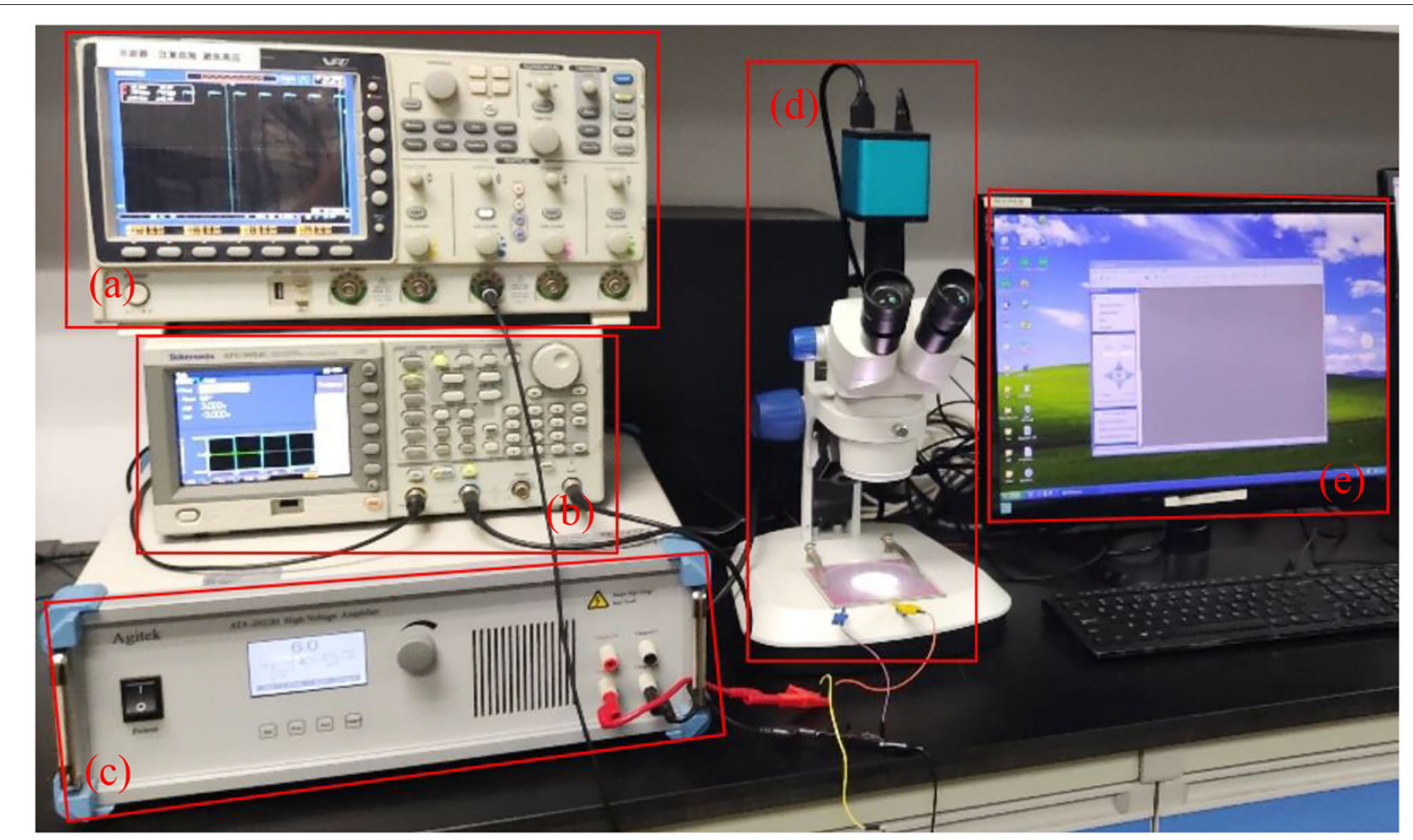

FIGURE 3 | Experimental platform for the testing of EWD contact angle hysteresis. It was composed of driving system and testing system. (a) Oscilloscope. (b) Function generator. (c) Amplifier. (d) Microscope. (e) Computer.

TABLE 1 | Parameters of the EWD panel.

\begin{tabular}{llcccc}
\hline $\begin{array}{l}\text { Panel size } \\
\left(\mathbf{c m}^{2}\right)\end{array}$ & Oil color & Resolution & $\begin{array}{c}\text { Pixel size } \\
\left(\mu \mathbf{m}^{2}\right)\end{array}$ & $\begin{array}{c}\text { Height of } \\
\text { pixel }(\mu \mathbf{m})\end{array}$ & $\begin{array}{c}\text { ITO layer } \\
(\mathbf{n m})\end{array}$ \\
\hline $10 \times 10$ & Blue & $320 \times 240$ & $150 \times 150$ & 18 & $\begin{array}{c}\text { Hydrophobic } \\
\text { layer } \\
\text { thickness }\end{array}$ \\
\hline
\end{tabular}

voltage sequence of the waveform was generated by the waveform generator, and the voltage was amplified by the amplifier. Then the output voltage of the amplifier was applied to an EWD panel. Finally, the video stream was captured by the highspeed camera combined with the microscope and displayed on another computer. The aperture ratio was calculated by a software.

The materials of an EWD panel used in the experiment are as follows. The material of hydrophobic insulating layer was Teflon AF1600, and the solution was a fluorocarbon solvent FC-43 of 3M Company in United States. Transparent polyimide was used as the grid material of pixel walls. Photoresist was SU-83005 which came from Microchem. The ITO glass substrate came from Shenzhen Laibao High-Tech Co., Ltd, with a thickness of $0.7 \mathrm{~mm}$ and an impedance of $100 \Omega / \mathrm{cm} 2$. The electrolyte solution was sodium chloride solution with a concentration of $1 \times 10-4 \mathrm{~mol} / \mathrm{L}$, deionized water was obtained by an ultra-pure ultraviolet water purification system. And the parameters of the EWD panel were listed in Table 1.

\section{Effect of Contact Angle Hysteresis on EWDs}

During the driving process of EWDs, the period that the driving voltage increases from $0 \mathrm{~V}$ to the maximum voltage and then decreases from the maximum voltage to $0 \mathrm{~V}$ is called a driving period $\mathrm{T}$. In $0-\mathrm{T} / 2$, the driving voltage can be gradually increased, and the pixel oil gradually shrinks from the flat state to a corner in a pixel. In the process from $T / 2$ to $T$, the oil state should be completely opposite to the first half cycle. In this case, the oil could be controlled more precisely and achieve a multiple gray-scale display.

However, the state of oil is different from an ideal situation. Two identical EWD panels were used to test the impact of contact angle hysteresis. The driving voltage was increased from 0 to $20 \mathrm{~V}$ and decreased from 30 to $20 \mathrm{~V}$ respectively. When starting voltages were different, the gray-scale obtained was different with a same target driving voltage. As shown in Figure 4, when the driving voltage was increased from 0 to $20 \mathrm{~V}$, the pixel aperture ratio was $24.85 \%$; when the driving voltage was decreased from 

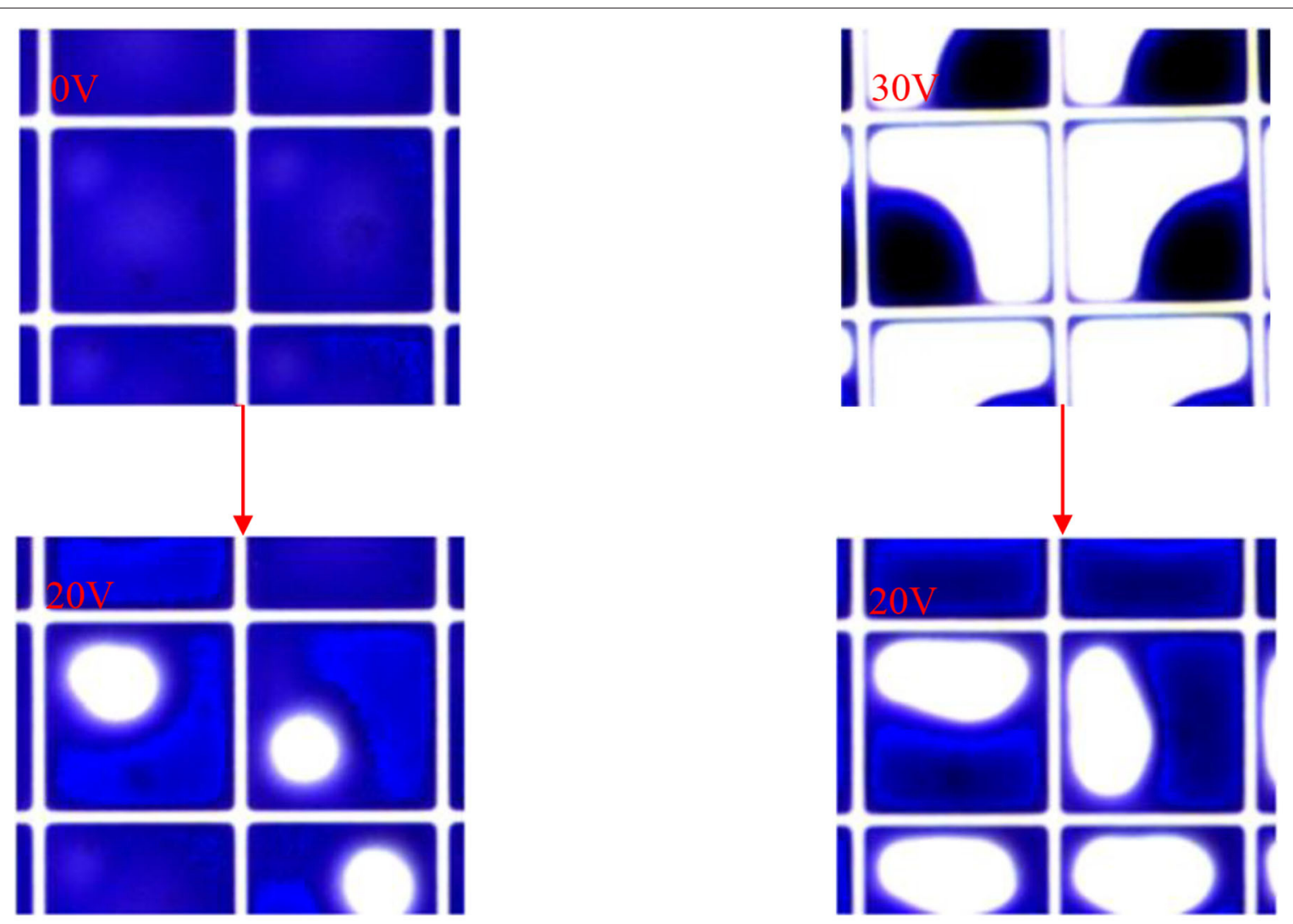

FIGURE 4 | Inconsistent performance of aperture ratio in a pixel. The driving voltage was increased from 0 to $20 \mathrm{~V}$ and decreased from 30 to $20 \mathrm{~V}$, respectively, the aperture ratio was different at $20 \mathrm{~V}$.

30 to $20 \mathrm{~V}$, the pixel aperture ratio was $40.12 \%$. The maximum difference of the aperture ratio was $15.27 \%$. During the process of driving voltage increased from 0 to $20 \mathrm{~V}$, the electric field force increased, and the oil was pushed to achieve a balance state. During the process of driving voltage decreased from 30 to $20 \mathrm{~V}$, the electric field force decreased, and the oil spread naturally due to the wetting effect. Due to the coarseness of hydrophobic insulation layer and the existence of charge trapping, the rising and falling process was asymmetric. This phenomenon is called hysteresis effect, and also can be called gray-scale distortion. The smaller the difference, the smaller the gray-scale distortion. This gray-scale distortion phenomenon was caused by contact angle hysteresis.

\section{Contact Angle Hysteresis in DC Driving Scheme and AC Driving Scheme}

In order to study the effect of contact angle hysteresis on aperture ratio of EWDs, some experiments were designed in this paper. The traditional square wave was selected to drive an EWD panel, and $60 \mathrm{~Hz}$ was determined as the default driving frequency [3]. As shown in Figure 5, they are square waves in DC driving scheme and AC driving scheme, respectively. The relationship between driving voltage and aperture ratio in DC driving scheme and AC driving scheme were tested.
During experiments, the overall trend of the pixel aperture ratio was increasing with the increasing of driving voltage. When the driving voltage was decreased, the aperture ratio was decreased, and finally returned to a flat state. When the voltage was increased from 0 to $30 \mathrm{~V}$, the oil state was in the forward process. When the voltage was decreased from 30 to $0 \mathrm{~V}$, the oil state was in the backward process. When the driving voltage was higher than $30 \mathrm{~V}$, the pixel could be broken down.

The test results are shown in Figure 6. Figure 6A shows the result of square wave in DC driving scheme, the range of aperture ratio was $1-61.48 \%$. At both ends of the driving voltage value, the distortion of the aperture ratio was small. When the driving voltage was in the middle stage, the rate of the aperture ratio distortion was bigger. Among these situations, when the driving voltage was $16 \mathrm{~V}$, the maximum distortion could reach to $35.82 \%$. During an entire EWD process, the scale distortion interval of gray-scale was $0-35.82 \%$. Figure 6B shows the result of square wave in $\mathrm{AC}$ driving scheme, the range of aperture ratio was $1-58.71 \%$. At both ends of the driving voltage value, the distortion of the aperture ratio was small. When the driving voltage was in the middle stage, the rate of the aperture ratio distortion was bigger. Among these situations, when the driving voltage was $13 \mathrm{~V}$, the maximum distortion could reach to $20.84 \%$. 


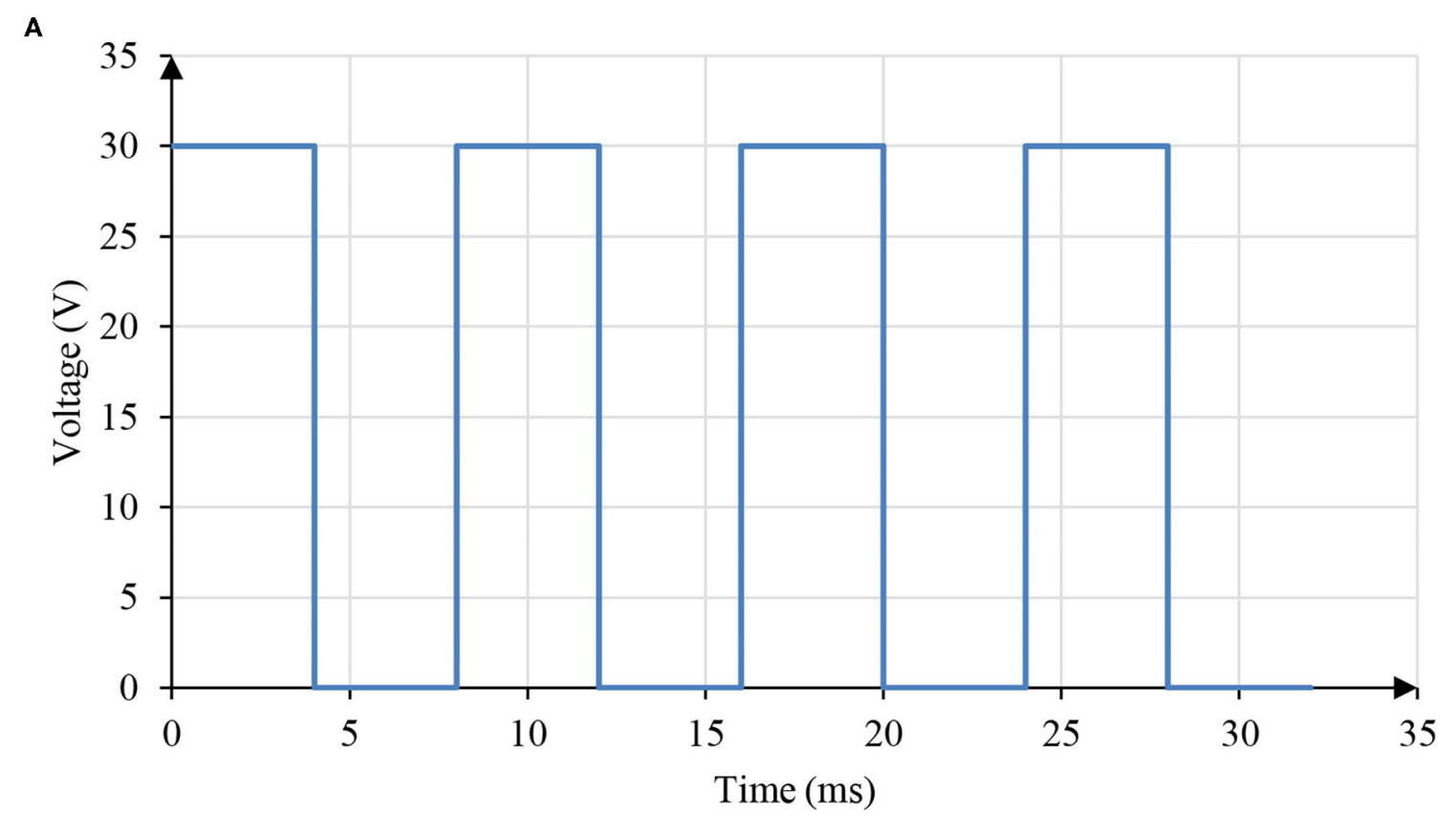

B

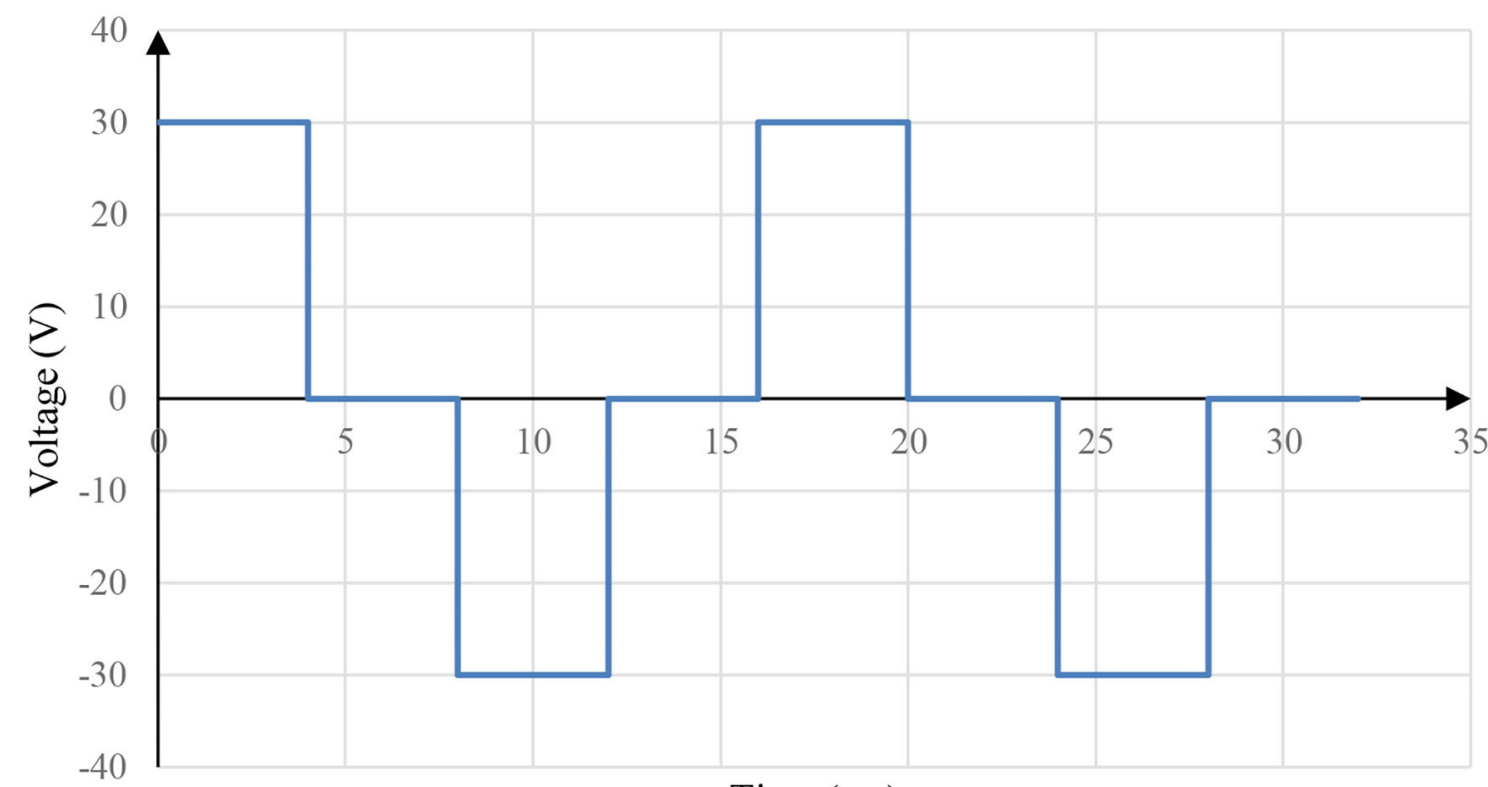

Time (ms)

FIGURE 5 | Square waves in DC driving scheme and AC driving scheme. (A) The square wave in DC driving scheme. (B) The square wave in AC driving scheme.

During an entire EWD process, the scale distortion interval of gray-scale was $0-20.84 \%$. It can be seen that the contact angle hysteresis of AC driving scheme is lower than that of DC driving scheme.
Frequency Optimization for the AC Driving Scheme

In order to determine the optimal driving frequency, more experiments were designed. The maximum voltage in $\mathrm{AC}$ driving 
A

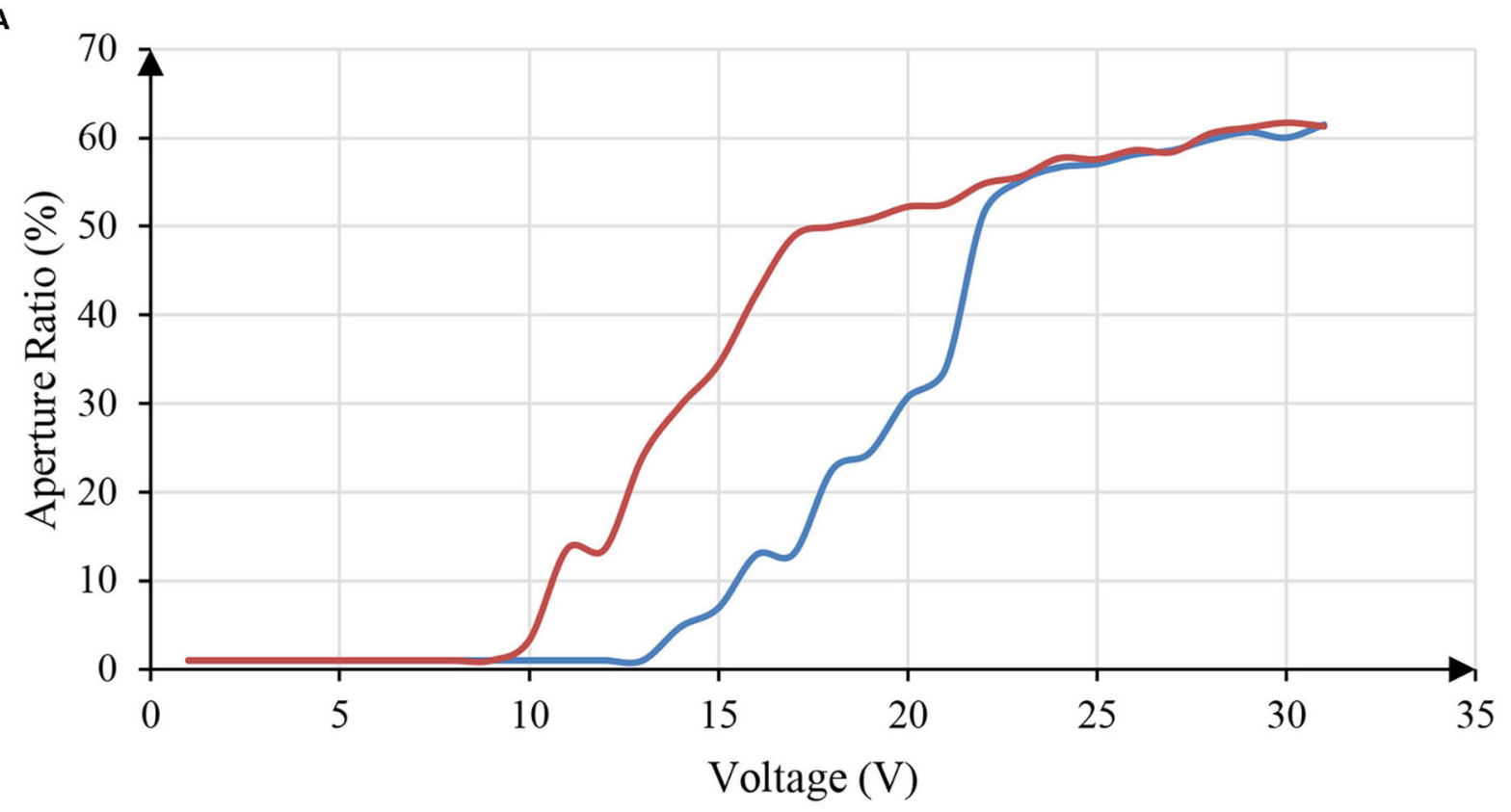

- Voltage Increase $\quad$ Voltage Decrease

B

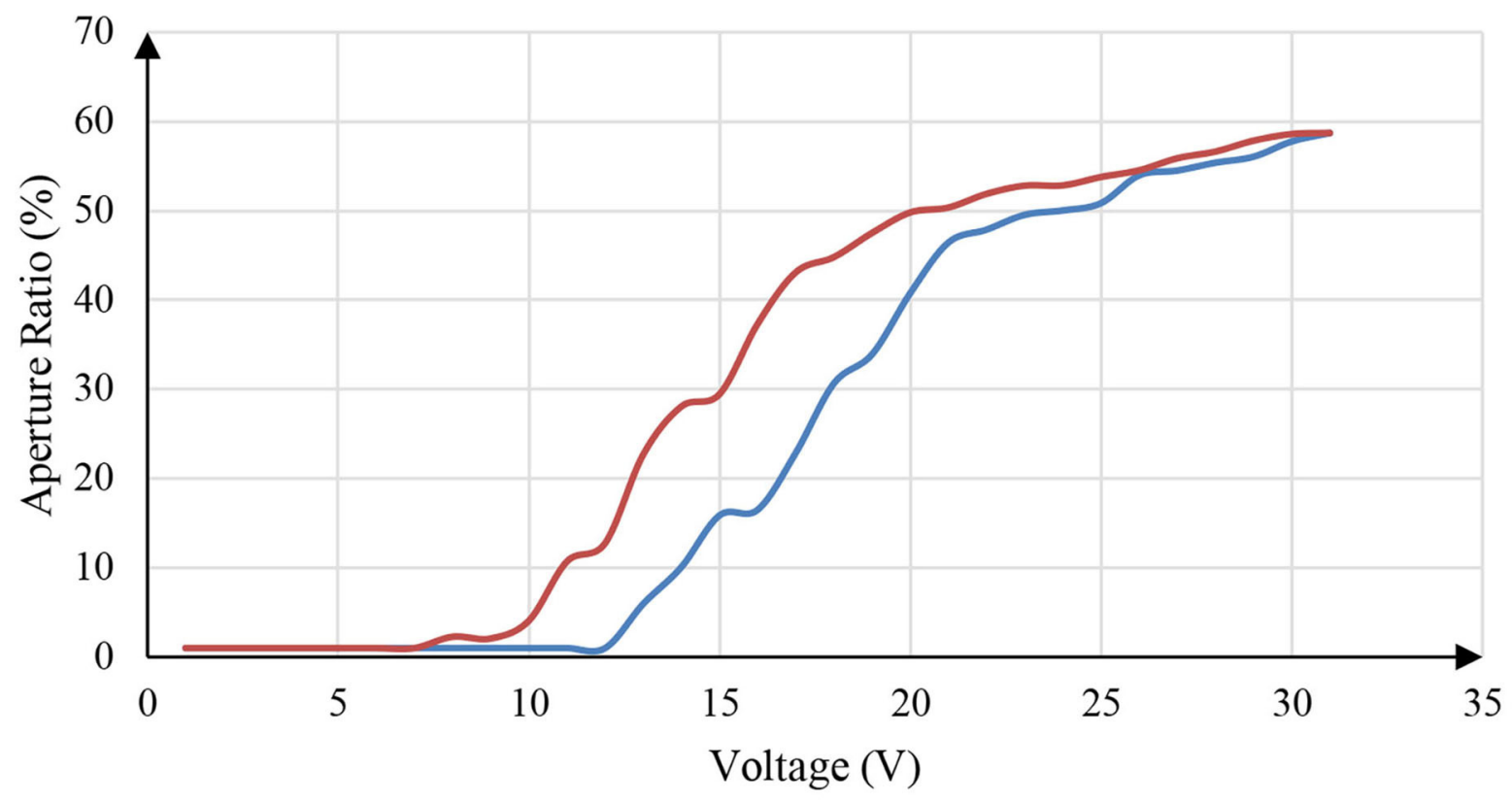

- Voltage Increase — Voltage Decrease

FIGURE 6 | Hysteresis curves of an EWD driven by DC driving scheme and AC driving scheme. The black curve showed the aperture ratio when driving voltage was increased from 0 to $30 \mathrm{~V}$, and the red curve showed the aperture ratio when driving voltage was decreased from 30 to $0 \mathrm{~V}$. (A) The hysteresis curve of an EWD driven by DC driving scheme. (B) The hysteresis curve of an EWD driven by AC driving scheme. 
scheme used in this paper was $30 \mathrm{~V}$. And AC voltages with frequency of $90-2,700 \mathrm{~Hz}$ were applied to EWD panels. Then the average value of aperture ratio was recorded, simulated and calculated by a microscope.

As shown in Figure 7, when the frequency was from 90 to $350 \mathrm{~Hz}$, the oil in pixels was unstable, and pixels were opened for a short time and closed in an instant. In addition, the unstable state of oil in the pixel led to an oil splitting. Although the driving voltage was remained unchanged, the aperture ratio of the pixel was decreased gradually with the passage of time, and the stability of the aperture ratio was insufficient.

When the frequency was $350-500 \mathrm{~Hz}$. The oil in pixels was stable, pixels were fully opened, the aperture ratio was $70.96 \%$, and pixels were hardly closed within $12 \mathrm{~s}$. When the frequency was $500-1,200 \mathrm{~Hz}$, some pixels were fully opened, and others were not fully opened. The oil state was not stable enough. The overall aperture ratio of pixels varied from 62.10 to $56.33 \%$. When the frequency was $1,200-2,700 \mathrm{~Hz}$, the oil state in pixels was stable, but pixels were not fully opened, and the aperture ratio was stable at about $51.89 \%$. At the same time, when the driving voltage was unchanged, the oil state had almost no change by time, and the aperture ratio was remained stable. When the driving voltage frequency was higher than $2,700 \mathrm{~Hz}$, as the frequency was changed, the aperture ratio was remained at about $50.73 \%$. Therefore, when the frequency was higher than $2,700 \mathrm{~Hz}$, the influence of driving voltage and frequency on aperture ratio was very small.

In the process of an $\mathrm{AC}$ voltage driving with a constant frequency, the oil movement path became more stable over time. We tested the stability of the aperture ratio within $12 \mathrm{~s}$ when the frequency was $90,175,350,470,670,1,200,1,700$, and 2,700 Hz. As shown in Figure 8.

It can be seen from Figure 8 that the aperture ratio reached the maximum with frequency of $90 \mathrm{~Hz}$. But the oil was gradually spread out from the fully contracted state within $2 \mathrm{~s}$. After $5 \mathrm{~s}$, the aperture ratio was decreased from about $69.76 \%$ to $50.77 \%$. In addition, during the oil shrinking process, the oil state was unstable and split. At the same time, the oil in pixels was driven randomly to four corners, and it could affect the display brightness of pixels. Compared with the frequency of $90 \mathrm{~Hz}$, the oil was more stable under the frequency of $175 \mathrm{~Hz}$. But within $1 \mathrm{~s}$, aperture ratio was decreased from 58.38 to

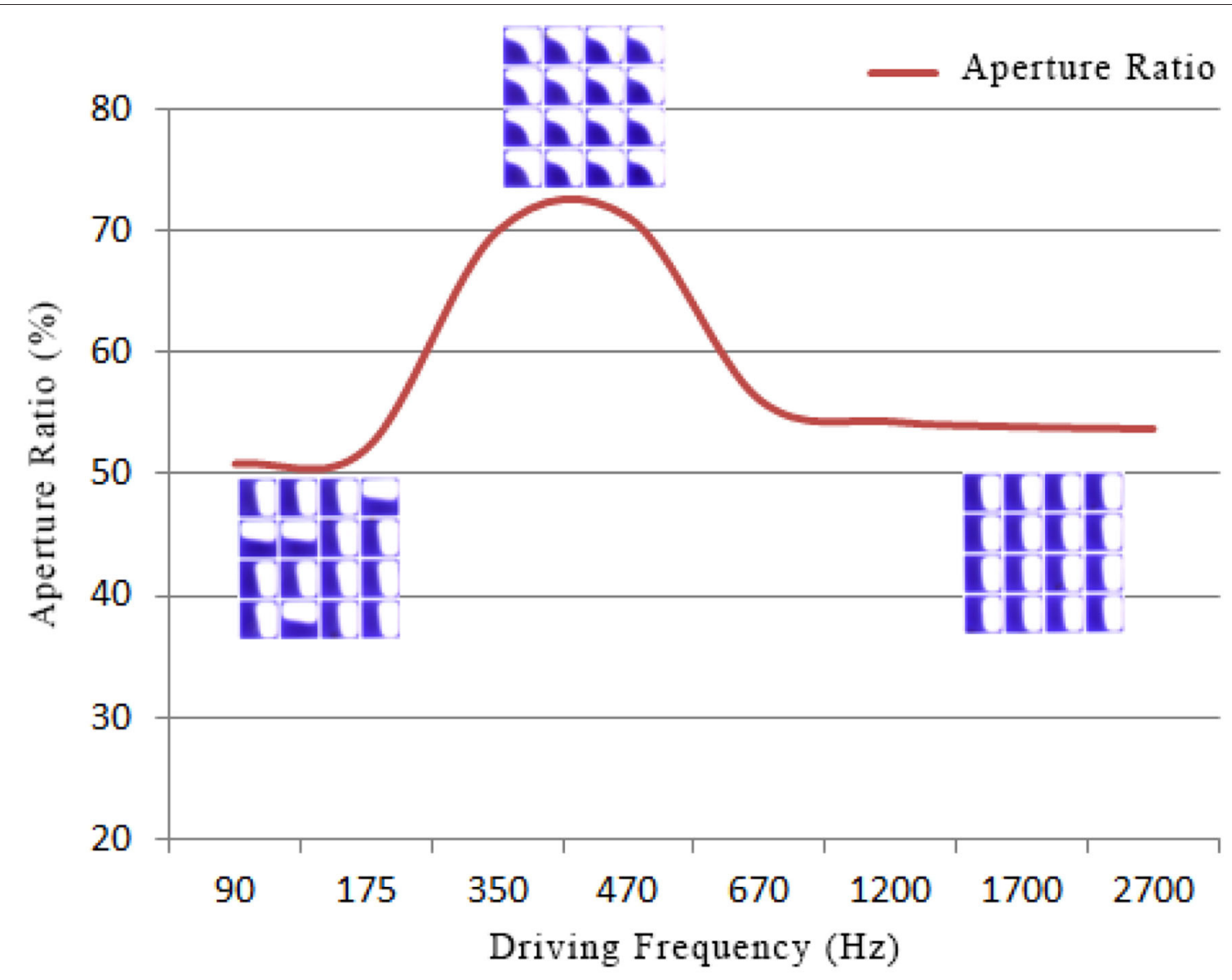

FIGURE 7 | The EWD was driven by a $30 \mathrm{~V}$ AC driving voltage, the aperture ratio changed when the frequency was increased from 90 to $2,700 \mathrm{~Hz}$. When the frequency was $350-500 \mathrm{~Hz}$, pixels were fully opened. 


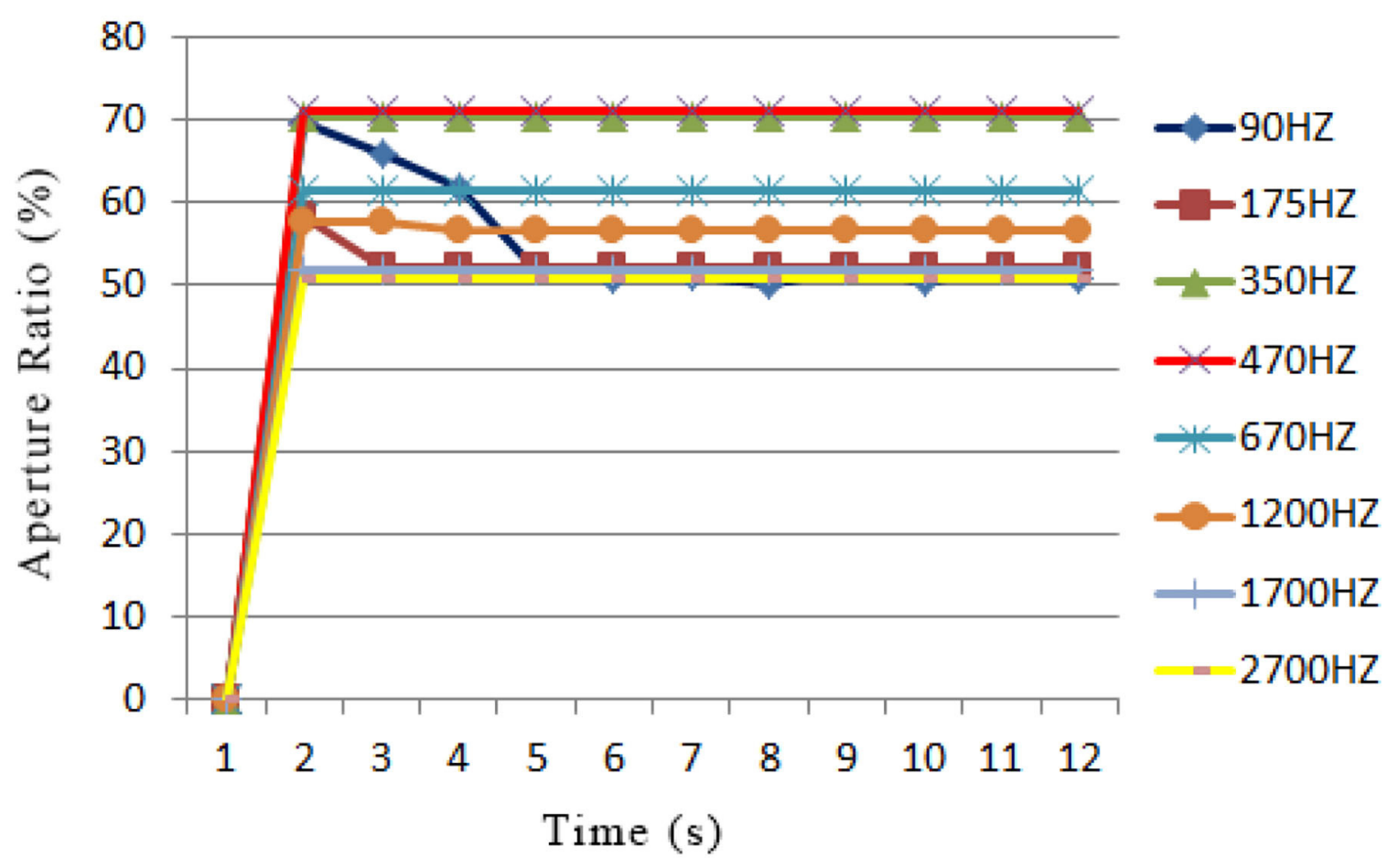

FIGURE 8 | The relationship between time and aperture ratio when an EWD was driven by a 30V AC driving voltage at different frequencies. The smoothness of curves represented the oil stability. The $470 \mathrm{~Hz}$ AC driving voltage had the largest aperture ratio and most stable aperture ratio.

$52.11 \%$. Then the aperture ratio was remained stable. When the frequency was higher than $350 \mathrm{~Hz}$, the stability of aperture ratio was significantly improved. When the frequency was 350 and $470 \mathrm{~Hz}$, the oil in pixels was completely contracted and remained stable within $12 \mathrm{~s}$. When the frequency was $670 \mathrm{~Hz}$, the aperture ratio was $61.33 \%$, and pixels were not fully opened. At this time, the aperture ratio was stable. When the frequency was $1,200,1,700$, and $2,700 \mathrm{~Hz}$, the aperture ratio and the oil state were stable, but pixels were not fully opened. Therefore, the $470 \mathrm{~Hz}$ AC driving voltage was selected in our driving scheme for EWDs.

The generation process of the $470 \mathrm{~Hz}$ AC driving voltage was as follows, the waveform generator can generate $470 \mathrm{~Hz}$ AC driving voltage with the maximum voltage difference of $10 \mathrm{~V}$. Therefore, the AC voltage was varied from +5 to $-5 \mathrm{~V}$. Then the output of waveform generator was amplified 6 times by a high voltage amplifier, so that the AC driving voltage can be changed from +30 to $-30 \mathrm{~V}$. During the $\mathrm{AC}$ driving process, the average value of the aperture ratio was calculated.

As shown in Figure 9, the red curve indicated that the driving voltage was increased from 10 to $25 \mathrm{~V}$ and the blue curve indicated that the driving voltage was decreased from 25 to $10 \mathrm{~V}$. When the voltage was $>25 \mathrm{~V}$ or $<10 \mathrm{~V}$, these two curves were coincident. In process (B), when the driving voltage was increased or decreased, the aperture ratio was basically the same, and the maximum difference between them was $3 \%$. In process (A), when the difference of aperture ratio was the largest, the EWD is shown in the Figure 10. Figure 10A showed the aperture ratio of EWDs during the driving voltage increasing process, the average aperture ratio was 26.50\%; Figure 10B showed the aperture ratio of EWDs during the driving voltage decreasing process, the average aperture ratio was $32.47 \%$. Therefore, the maximum difference between them is $5.97 \%$. When the traditional DC voltage was used, the maximum distortion was $35.82 \%$. Although the distortion could not be completely eliminated, the aperture ratio distortion could be reduced by $29.85 \%$ with the AC driving scheme.

\section{CONCLUSIONS}

In order to reduce the distortion of aperture ratio and achieve precise control of gray-scales, an AC driving scheme was proposed for improving the performance of EWDs. This scheme can effectively reduce the influence of contact angle hysteresis. The advantages and disadvantages of AC driving voltages at different frequencies were analyzed by experiments. And then, according to the stability and aperture ratio of EWDs, a $470 \mathrm{~Hz}$ AC driving scheme was determined. Compared with traditional DC driving scheme, the aperture ratio distortion caused by contact angle hysteresis could be reduced to $5.97 \%$. Therefore, this scheme could be applied 


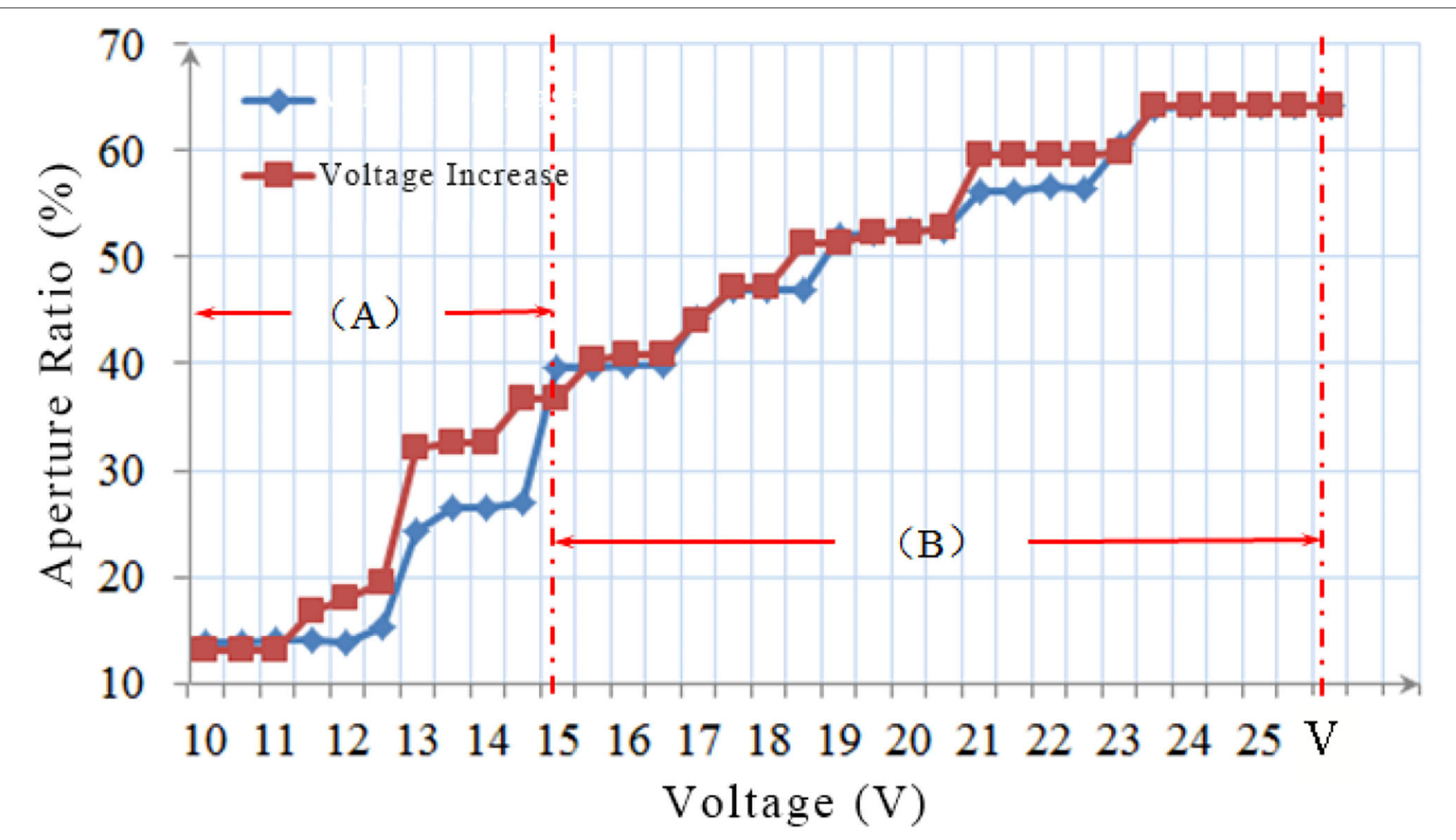

FIGURE 9 | Hysteresis characteristic curves of an EWD driven by a $470 \mathrm{~Hz}$ AC driving voltage. The red curve represented the aperture ratio when the driving voltage was increased from 10 to $25 \mathrm{~V}$, the blue curve represented the aperture ratio when the driving voltage was decreased from 25 to $10 \mathrm{~V}$. The maximum distortion is $5.97 \%$. (A) Indicates the state where the difference of aperture ratio changes greatly, (B) indicates the stage where the difference of aperture ratio changes little.

\section{A}

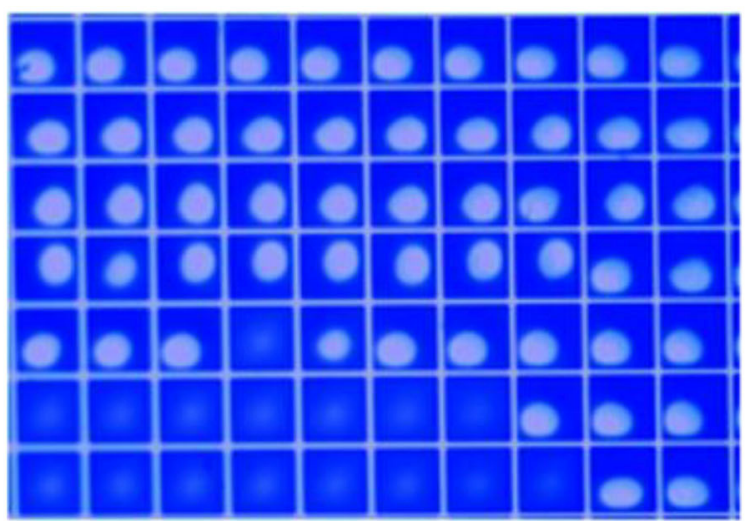

B

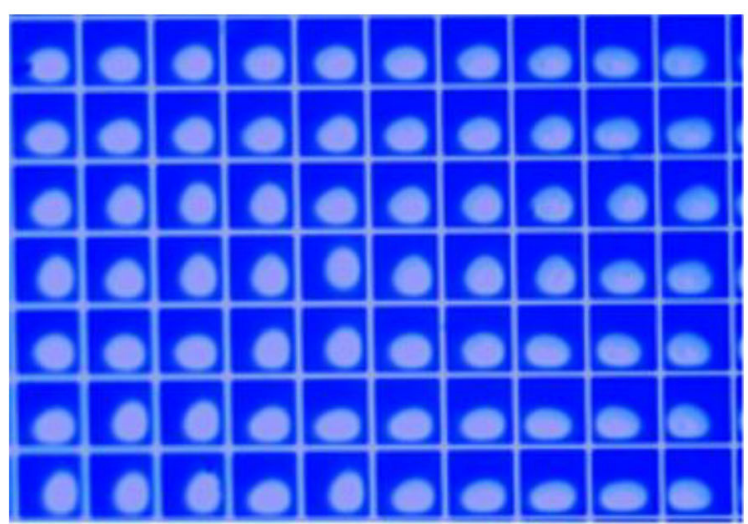

FIGURE 10 | The aperture ratio of pixels when the EWD was driven by $470 \mathrm{~Hz}$ AC driving voltage. (A) The pixel aperture ratio was $26.50 \%$ during the voltage increasing process. (B) The pixel aperture ratio was $32.47 \%$ during the voltage decreasing process. The maximum difference was $5.97 \%$.

to EWDs system to achieve more accurate control for displaying gray-scales.

\section{DATA AVAILABILITY STATEMENT}

The raw data supporting the conclusions of this article will be made available by the authors, without undue reservation.

\section{AUTHOR CONTRIBUTIONS}

LW and WL designed this project. LW and HZ carried out most of the experiments and data analysis. JL and ZY carried out a few of the experiments and data analysis. WL, $\mathrm{HZ}, \mathrm{WL}, \mathrm{JL}, \mathrm{ZY}, \mathrm{QW}, \mathrm{JZ}$, and PM gave suggestions on the project management and conducted helpful discussion on the 
experimental results. All authors contributed to the article and approved the submitted version.

\section{FUNDING}

This research was supported by the Special Projects in Key Fields of Colleges and Universities of Guangdong in 2020 (No. 2020ZDZX3083), the Second Batch of Social Welfare and Basic

\section{REFERENCES}

1. Heikenfeld J, Drzaic P, Yeo J S, Koch T. Review paper: a critical review of the present and future prospects for electronic paper. J Soc Inform Display. (2011) 19:129-56. doi: 10.1889/JSID19.2.129

2. Mingyong Q, Shanling L, Suyun Z, Zhixian L, Tailiang G, Biao T. Realtime dynamic driving system implementation of electrowetting display. OptoElectronic Eng. (2019) 46:180623. doi: 10.12086/oee.2019.180623

3. Li W, Wang L, Zhang T, Lai S, Liu L, He W, et al. Driving waveform design with rising gradient and sawtooth wave of electrowetting displays for ultra-low power consumption. Micromachines. (2020) 11:145. doi: 10.3390/mi11020145

4. Heikenfeld J, Zhou K, Kreit E, Raj B, Yang S, Sun B, et al. Electrofluidic displays using young-laplace transposition of brilliant pigment dispersions. Nat Photo. (2009) 3:292-6. doi: 10.1038/nphoton.2009.68

5. Tröls A, Enser H, Jakoby B. Modeling and fabrication of low-cost electrowetting actuators for flexible microfluidic display applications. 2016 IEEE Sens. (2016) 10:1-3. doi: 10.1109/ICSENS.2016.7808429

6. Zhao R, Cumby B, Russell A, Heikenfeld J. Large area and low power dielectrowetting optical shutter with local deterministic fluid film breakup. Appl Phys Lett. (2013) 103:223510. doi: 10.1063/1.4834095

7. Beni G, Hackwood S. Electro-wetting displays. Appl Phys Lett. (1981) 38:2079. doi: 10.1063/1.92322

8. Hayes RA, Feenstra BJ. Video-speed electronic paper based on electrowetting. Nature. (2003) 425:383-5. doi: 10.1038/nature01988

9. $\mathrm{Xu} \mathrm{ZN}$. An algorithm for selecting the most accurate protocol for contact angle measurement by drop shape analysis. Rev Sci Instrum. (2014) 85:125107. doi: 10.1063/1.4903198

10. Yi Z, Huang Z, Lai S, He W, Wang L, Chi F, et al. Driving waveform design of electrowetting displays based on an exponential function for a stable grayscale and a short driving time. Micromachines. (2020) 11: 313. doi: 10.3390/mi11030313

11. Yi Z, Liu L, Wang L, Li W, Shui L, Zhou G. A driving system for fast and precise gray-scale response based on amplitude-frequency mixed modulation in TFT electrowetting displays. Micromachines. (2019) 10:732. doi: 10.3390/mi10110732
Research Key Projects of Zhongshan (No. 2020B2021), the School Level Project of Zhongshan Polytechnic (No. 2018KQ22), the Key Technology Development of High Frequency Antenna Substrate Based on 5G Communication (No. 2020ZDZX2081), Key Project of Provincial Education Department, the Key Technology Research and Development of High Frequency Substrate Based on 5G Communication (No. KYA2002), and Key Scientific Research Project of the University.

12. Li F, Mugele F. How to make sticky surfaces slippery: contact angle hysteresis in electrowetting with alternating voltage. Appl Phys Lett. (2008) 92:244108. doi: 10.1063/1.2945803

13. Giraldo A, Aubert J, Bergeron N, Li F, Slack A, Weijer M. Transmissive electrowetting-based displays for portable multi-media devices. Sid Sympos Digest Techn Papers. (2012) 40:479-82. doi: 10.1889/1.32 56820

14. Yi Z, Feng W, Wang L, Liu L, Lin Y, He W, et al. Aperture ratio improvement by optimizing the voltage slope and reverse pulse in the driving waveform for electrowetting displays. Micromachines. (2019) 10:862. doi: 10.3390/mi101 20862

15. Van Dijk R, Feenstra BJ, Hayes RA, Camps IGJ, Boom RGH, Wagemans $\mathrm{MMH}$, et al. Gray scales for video applications on electrowetting displays. Sid Sympos Digest Techn Papers. (2006) 37:1926-9. doi: 10.1889/1.2433427

16. Seveno D, Blake TD, Coninck JD. Young's equation at the nanoscale. Phys Rev Lett. (2013) 111:096101. doi: 10.1103/PhysRevLett.111.096101

17. Dou Y, Wang B, Jin M, Yu Y, Zhou G, Shui L. A review on self-assembly in microfluidic devices. J Micromech Microeng. (2017) 27:113002. doi: 10.1088/1361-6439/aa84db

18. Chen Z, Lin S, Lin Z, Liao Q, Li T, Tang B. Design of video display driving system for low-power electrowetting display. Acta Photo Sinica. (2020) 49:222002. doi: 10.3788/gzxb20204902.02 22002

Conflict of Interest: The authors declare that the research was conducted in the absence of any commercial or financial relationships that could be construed as a potential conflict of interest.

Copyright (c) 2021 Wang, Zhang, Li, Li, Yi, Wan, Zhang and Ma. This is an openaccess article distributed under the terms of the Creative Commons Attribution License (CC BY). The use, distribution or reproduction in other forums is permitted, provided the original author(s) and the copyright owner(s) are credited and that the original publication in this journal is cited, in accordance with accepted academic practice. No use, distribution or reproduction is permitted which does not comply with these terms. 